\title{
Étude sur le Triangle et sur certains points de Géométrographie.
}

\section{Par M. Émile Lemoine.}

Cette note que j'ai l'honneur de prèsenter à la Société Mathématique d'Edinburgh, par l'entremise aimable de M. J. S. Mackay, contient, ou des résultats que je crois nouveaux, ou des développements sur des sujets que j'ai déjà souvent abordés dans la Géométrie et qui concernent: la transfurmation continue dans le triangle et dans le tétraèdre, les formules entre les eléments du triangle, et la Géométrographie. Pour abréger, je passerai rapidement sur les points que j'ai déjà développés ailleurs, me contentant de renvoyer, si l'on désire plus d'explications, aux mémoires où la chose a été faite.

L'idée de la transformation continue n'est autre qu'une explicitation, pour le triangle et pour le tétraèdre, du principe de continuité de Carnot; elle permet de transformer les théorèmes, les formules, les équations qui déterminent les éléments de ces figures ou expriment leurs propriétés, de façon à ce que, sous le nouveau vêtement qu'elle leur fait prendre, on obtient des théorèmes, des formules, des équations nouvelles, mais qui ne sont, au fond, que des formes d'une même vérité.

Notations pour le triangle $\mathrm{ABC}$ et ses éléments.

Nous nommons $a, l, c ; A, \mathrm{~B}, \mathrm{C} ; p, p-a, p-b, p-c ; \mathrm{S} ; \mathrm{R}$; $r, r_{a}, r_{b}, r_{c} ; \delta, \delta_{a}, \delta_{b}, \delta_{c} ; \omega ; h_{a}, l_{b}, h_{c} ; l_{a}, l_{b}, l_{c} ; l_{a}^{\prime}, l_{b}^{\prime}, l_{c}^{\prime}$ respentivement les trois côtés $\mathrm{BC}, \mathrm{CA}, \mathrm{AB}$; les trois angles; les quantités $\frac{1}{2}(a+b+c), \frac{1}{2}(b+c-a), \frac{1}{2}(c+a-b), \quad \frac{1}{2}(a+b-c) ;$ la surface; le rayon du cercle circonscrit ; les rayons du cercle inscrit et des trois cercles ex-inscrits; les quantités $4 R+r, 4 R-r_{b}, 4 R-r_{b}, 4 R-r_{c}$; l'angle de Brocard; les trois hauteurs; les trois bissectrices intérieures; les trois bissectrices extérieures.

J'appelle $x, y, z$ les coordonnées normales trilinéaires, par rapport au triangle de référence $A B C$.

J'appelle $a, \beta, \gamma$ les coordonnées barycentriques par rapport à ce même triangle; et $\mathrm{X}, \mathrm{Y}$ les coordonnées cartésiennes, quels qu' soient les axes de coordonnées et leur angle.

Voici un tableau qui donne le moyen d'opérer dins tous les cas, la transformation continue soit en A, soit en B, soit en C, dans les théorèmes, les formules, les équations. 


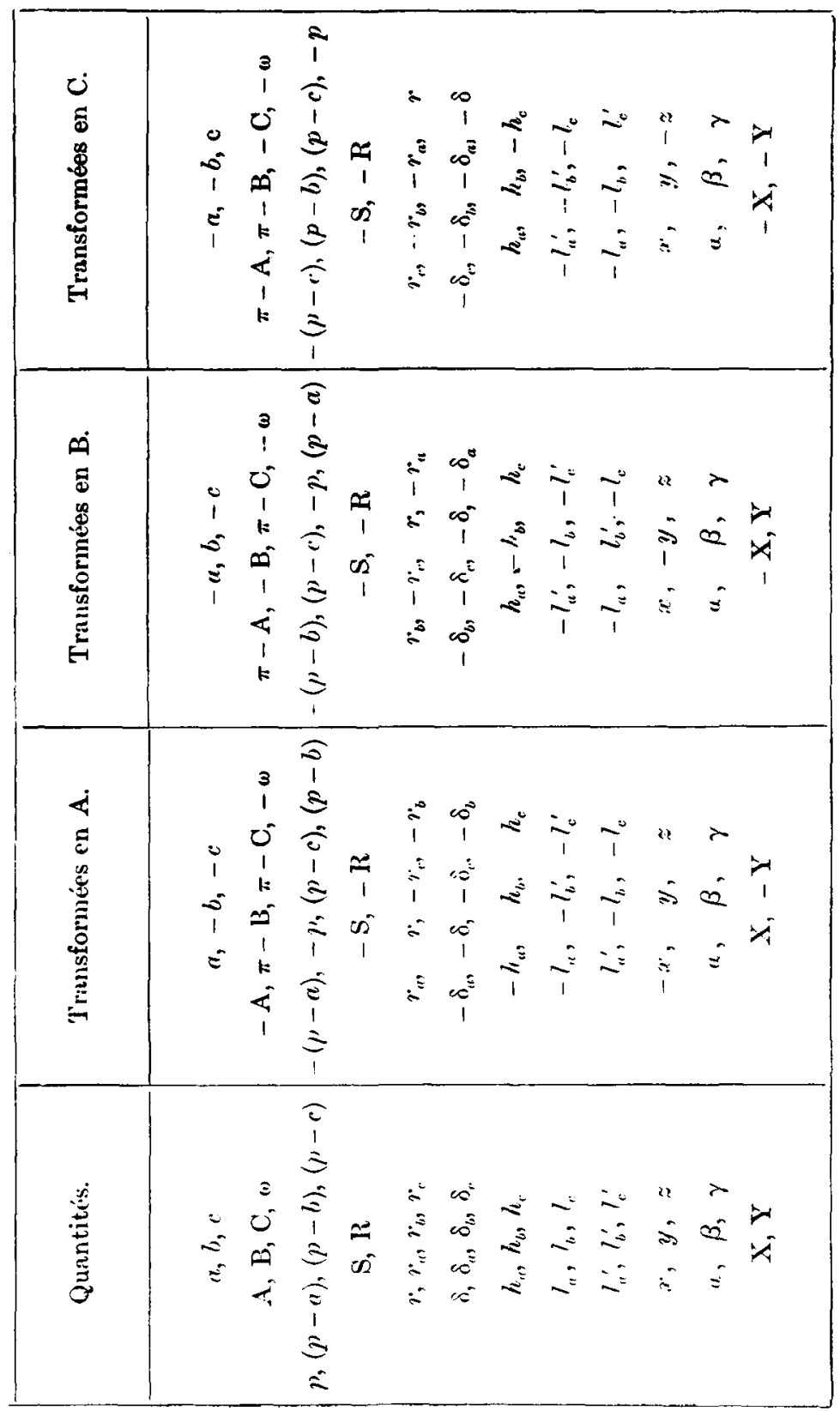


$\mathrm{Si}$, dans une formule ou dans une équation se rapportant au triangle $\mathrm{ABC}$ et contenant les éléments marqués dans la première colonne de ce tableau indiquée par le mot: quantité, je remplace ces éléments par ceux qui leur correspondent dans une des trois colonnes suivantes, j'aurai opéré respectivement la transformation continue en $\mathbf{A}$, en $\mathbf{B}$, ou en $\mathbf{C}$.

\section{Dans une transformation continue:}

I. La droite de l'intini se transforme en elle-même; donc, deux droites parallèles restent parallèles dans la figure transformée.

II. Les points circulaires de l'infini se transforment l'un dans l'autre.

III. Le degré et la classe des courbes se conservent dans les courbes transformées.

IV. L'homographie, l'homologie, et l'orthologie ainsi que l'involution, se conservent.

V. Deux droites ou deux courbes qui sont orthogonales restent orthogonales.

VI. Si les longueurs de cleux droites sont dins un rapport numérique indépendant des éléments du triangle, ce rapport se conservera.

La transformation continue faite en $\mathrm{A}$, en $\mathrm{B}$, et en $\mathrm{C}$ successivement présente les quatre cas suivants:

a. Elle ne change rien à la formule, à l'equation, au théorìme que l'on considère, qui se reproduit.

Exemples : $\quad a \cos \mathrm{B}+b \cos \mathrm{A}=c$.

Les trois hauteurs d'un triangle se coupent au même point dont les coordonnées sont

$$
\frac{1}{\cos A}, \frac{1}{\cos B}, \frac{1}{\cos C}
$$


b. A chacune des trois transformations correspond un résultat différent.

Exemples: Au point qui a pour coordonnées

$$
\frac{p-a}{a}, \frac{p-b}{b}, \frac{p-c}{c} \text { (point de Nagel) }
$$

correspondent respectivement les trois points :

$-\frac{p}{a}, \frac{p-c}{b}, \frac{p-b}{c} ; \quad \frac{p-c}{a},-\frac{p}{b}, \frac{p-a}{c}$

$\frac{p-b}{a}, \frac{p-a}{b},-\frac{p}{b}$, avec des propriétés analogues à celles du point de Nagel.

L'axe anti-orthique (droite qui passe par les pieds des trois bissectrices extérieures et a pour équation $x+y+z=0$ ) devient par transformation continue en $A$, la droite qui passe par les pieds des bissectrices intérieures partant de B et de $\mathrm{C}$ et par le pied de la bissectrice extérieure partant de $\mathrm{A}$; son équation est $-x+y+z=0$. On appelle souvent cette droite l'interbissectrice relative à $A$. Résultats analogues par transformation en $B$ et en $C$.

c. Une des transformations conserve le résultat primitif, les deux autres le changent en un autve résultat, mais unique pour ces deux transformations.

Exemple : La fornule $\mathrm{S}=\frac{a r_{b} r_{e}}{\delta-r_{a}}$ se reproduit par transformation en $A$, mais transformée soit en $B$, soit en $C$, elle donne

$$
\mathrm{S}=\frac{a r r_{a}}{4 \mathrm{R}-r_{b}-r_{c}}=\frac{a r r_{a}}{r_{a}-r}
$$

d. La transformation continue faite soit en $\mathrm{A}$, soit en $\mathrm{B}$, soit en $\mathrm{C}$ donne un méme résultat différent de celui qu'on transforme. 
Exemple: La conique inscrite qui a pour un cle ses foyers le point d'aù l'on voit les trois côtés sous le noême angle (Conique de Simmons : voir J. J. Milne, Companion to the Weekly Problem Papers, p. 165), conique qui a pour équation

$$
\sqrt{x \sin \left(\mathrm{A}+60^{\circ}\right)}+\sqrt{y \sin \left(\mathrm{B}+60^{\circ}\right)}+\sqrt{z \sin \left(\mathrm{C}+60^{\circ}\right)}=0,
$$

se transforme soit en $A$, soit en $B$, soit en $C$, en

$$
\sqrt{x \sin \left(\mathrm{A}-60^{\circ}\right)}+\sqrt{y \sin \left(\mathrm{B}-60^{\circ}\right)}+\sqrt{z \sin \left(\mathrm{C}-60^{\circ}\right)}=0 .
$$

Je n'ai pas trouvé de cas où l'une des transformations reproduise la formule et oi les deux autres la modifient mais chacune d'une façon différente.

La transformation continue s'applique au tétraèdre. Nous allons donner seulement le tableau que permet la transformation des éléments de la figure.

\section{Notatrons.}

1. Je clésigne par A, B, C, D les sommets du tétraèdr\%.

$2^{\circ}$. Les faces ABC, BCD, CDA, DAB seront $\mathrm{F}_{d}, \mathrm{~F}_{a}, \mathrm{~F}_{b}, \mathrm{~F}_{r}$, nous poserons $\mathrm{F}_{c}+\mathrm{F}_{b}+\mathrm{F}_{\mathrm{c}}+\mathrm{F}_{c}=\mathrm{S}$.

$3^{\circ}$. Les angles plans des faces

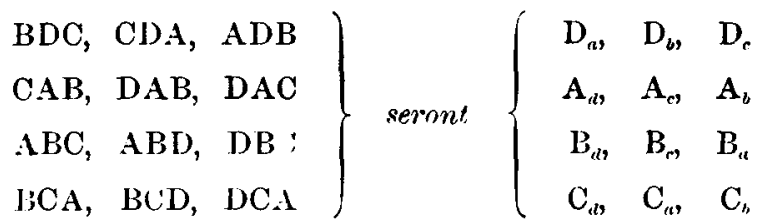

4. Les longueurs des ôtés $\mathrm{BC}, \mathrm{CA}, \mathrm{AB}, \mathrm{DA}, \mathrm{DB}, \mathrm{DC}$ seront "a, b, c, a', l', '’.

$5^{\circ}$. Les angles dièdres qui ont pour arêtes $\mathrm{BC}, \mathrm{CA}, \mathrm{AB}$, DA, DB, DC seront $\widehat{a}, \widehat{b}, \widehat{c}, \widehat{a^{\prime}}, \widehat{b}^{\prime}, \widehat{c^{\prime}}$.

Les angles que fait une arête avec les deux faces qui ne la contiennent pas seront désignés chacun par le lettre qui représente l'arête, suivie de la lettre qui représente la face considérée. 
Il y aura donc les douze angles

$$
\begin{aligned}
& \widehat{a \hat{\mathrm{F}}_{c}}, \quad \widehat{a \mathrm{~F}}_{b}, \quad \widehat{a}^{\prime} \mathrm{F}_{d}, \quad \widehat{a}^{\prime} \mathrm{F}_{a} \\
& \widehat{b} \widehat{\mathrm{F}}_{c}, \quad \widehat{b \mathrm{~F}_{a}}, \quad b \widehat{\mathrm{F}}_{d}, \quad \widehat{b}_{b} \\
& \widehat{c F}_{b}, \quad \widehat{\mathrm{F}}_{a}, \quad \widehat{c}^{\prime} \mathrm{F}_{l l}, \quad \widehat{c}^{\prime} \mathrm{F}_{c}
\end{aligned}
$$

$6^{\circ}$. Les lunuteurs seront: $h_{a}, h_{b}, h_{c}, h_{d}$.

$7^{\circ}$. Les rayons et les centres de la sphère inscrite et des sphères ex-inscrites de PREMİRE ESPÈCE seront:

$$
r, r_{w}, r_{b}, r_{c}, r_{c l} ; o, o_{a}, o_{b}, o_{c}, o_{d} \text {. }
$$

Les rayons et les centres des sphères ex-inscrites de SECONDE ESPÈCE, ou splières inscrites dans les combles du tétraèdre seront

$$
r_{a}{ }^{\prime}, r_{b}{ }^{\prime}, r_{c}^{\prime} ; o_{a}^{\prime}, o_{b}{ }^{\prime}, o_{c}^{\prime},
$$

$r_{a}{ }^{\prime}$ et $o_{a}{ }^{\prime} ; r_{b}{ }^{\prime}$ et $o_{b}{ }^{\prime} ; r_{c}{ }^{\prime}$ et $o_{c}^{\prime}$ appartenant respectivement à la sphère inscrite dans l'un des combles qui ont pour arêtes DA ou BC; $\mathrm{DB}$ ou $\mathrm{CA}$; $\mathrm{DC}$ ou $\mathrm{AB}$.

(On sait qu'il n'y a qu'une seule sphère pour deux combles opposés.)

Le tétraèdre général possède toujours ces huit sphères tangentes aux quatre faces; quand une sphère ou deux sphères, ou les trois sphères inscrites dans les combles manquent, ce qui peut arriver, car lorsque la somme de deux faces qui ont même arête est égale à la somme des deux autres, la sphère des combles correspondant à ces arêtes a un rayon infini; ce ne sont plus alors des tétraèdres généraux puisqu' il y a une ou plusieurs relations entre les faces, et la transfurmation continue n'est plus applicable, au moins sans discussion préalable.

$8^{\circ}$. Le volume du tétraèdre et le rayon de la sphère circonscrite seront: $\mathrm{V}$ et $\mathrm{R}$.

$O$ sera le centre de cette sphère.

$9^{\circ}$. Les angles de $\mathrm{DA}$ avec $\mathrm{BC}$; de $\mathrm{DB}$ et de $\mathrm{AC} ;$ de $\mathrm{DC}$ et de BA seront : $a, \beta, \gamma$.

$10^{\circ}$. Les longueurs des droites qui joignent les milieux de $\mathrm{DA}$ et de $\mathrm{BC}$; de $\mathrm{DB}$ et de $\mathrm{AC}$ de $\mathrm{DC}$ et de $\mathrm{BA}$ seront : $l, m, n$. 
De même que la transformation continue en $A$, duns le triangle, revient à changer $a, b, c$ en $a,-b,-c$, la transformation continue en $\mathrm{D}$, dans le tétraèdre, revient à changer $a, b, c, a^{\prime}, b^{\prime}, c^{\prime}$ en $a, b, c,-a^{\prime},-b^{\prime},-c^{\prime}$.

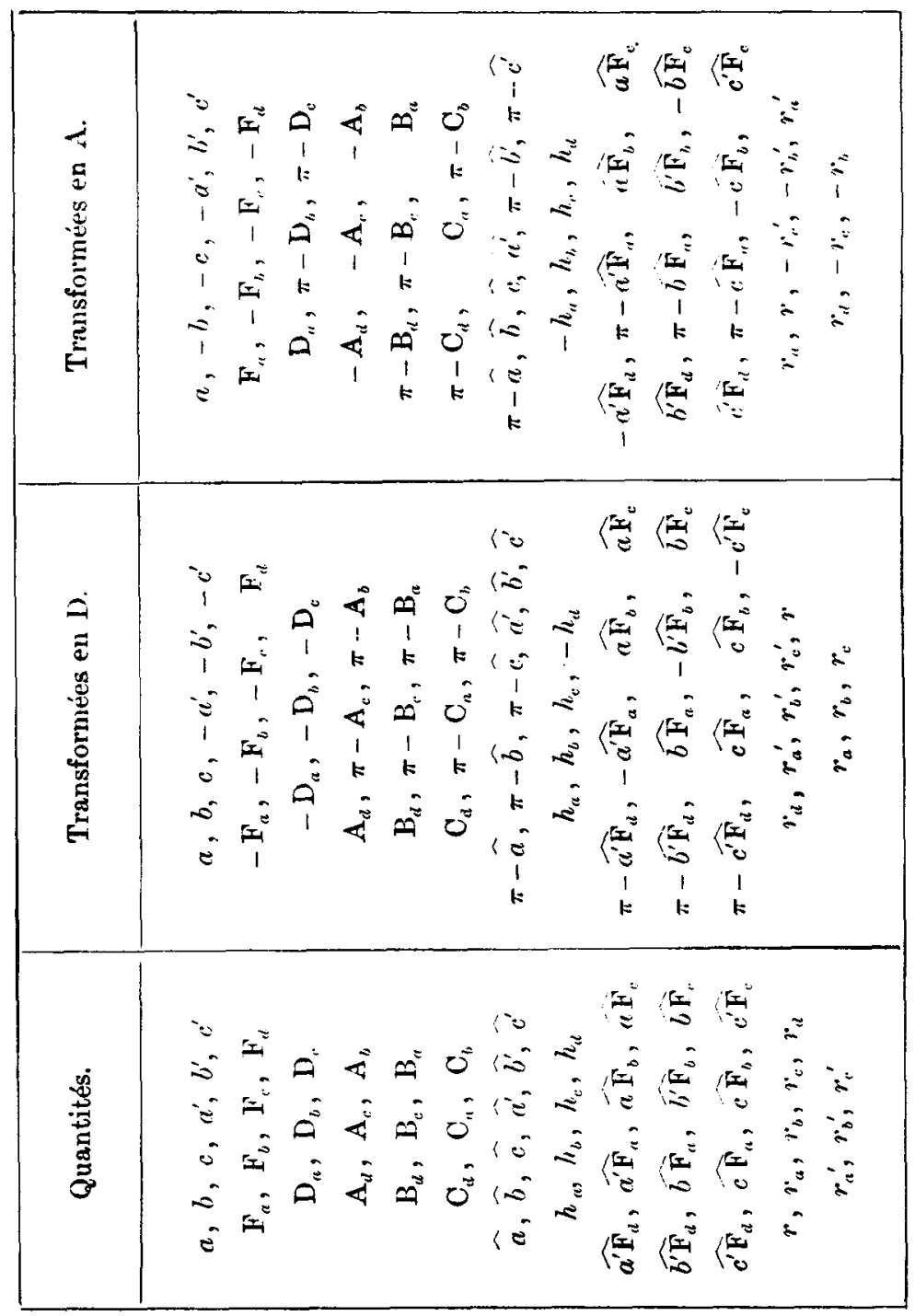




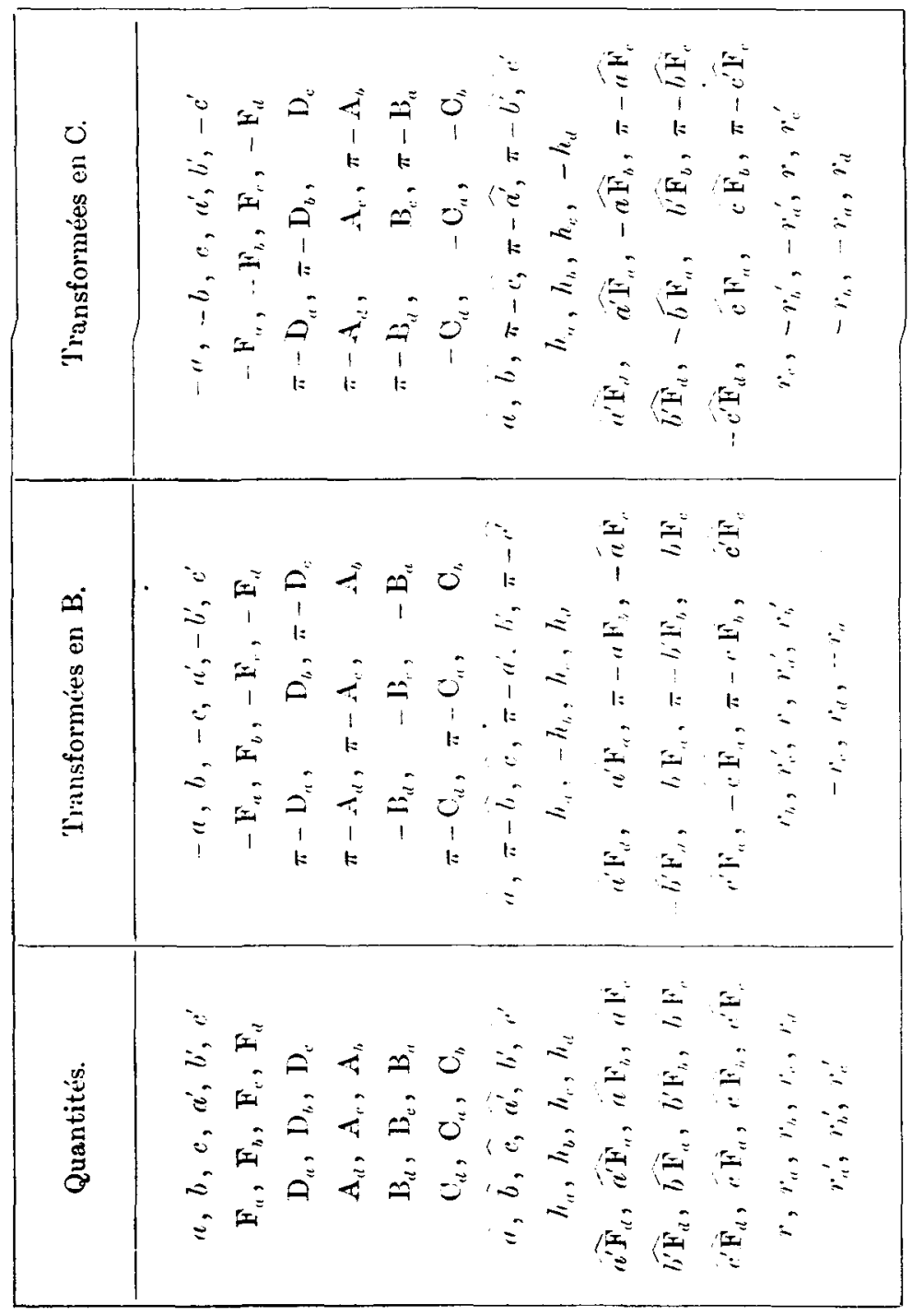

$\mathrm{V}$ et $\mathrm{R}$ se changent en : $-\mathrm{V}$ et $-\mathrm{R}$ dans les quatre transformations.

$a, \beta ; \gamma$ deviennent : $\pi-a, \pi-\beta, \pi-\gamma$ clans les quatre transformations.

$l, m, n$ ne changent pas. 
Nous avons ru pil le tableiu relatif au triangle qu'à un point donné $\mathbf{M}(x, y, z)$ dans le plan du triangle peuvent correspondre trois transformés continus $\mathbf{M}_{a}, \mathbf{M}_{b}, \mathbf{M}_{c}$ dont les coordonnées sont

$$
-x_{a}, y_{b}, z_{a} ; x_{b},-y_{b}, z_{b} ; x_{r}, y_{c},-z_{c}
$$

en affectant des indices $a, b, c$ les quantités qui représentent ce que deviennent les coordonnées quand on les transforme en $\mathrm{A}$, en $\mathrm{B}$, et en C. Par rapport au tétmèdre un point $\mathbf{M}(x, y, \approx, t)$ peut avoir sept transformés continus dont les coordonnées sont

$1^{\circ}$. Quatre transformés de premiere espèce

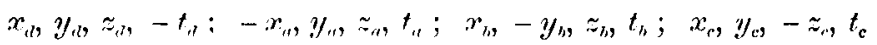

$2^{\circ}$. Trois transformés de seconde espíce

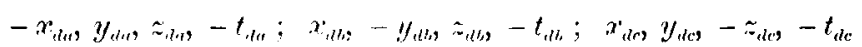

$x_{c}$ désignant ce que devient $x$ par transformation continue en $D$, $x_{d a}$ désignant ce que devient $x$ si l'on fait d'abord la transformation continue en D sur lui, ce qui donme $x_{d}$; puis la transformation continue en $d$ sur $x_{t l}$, ce qui donne $x_{l l a}$, etc.

Pour les démonstrations et l'exposition de la transformation continue nous renvoyons aux mémoires suivants: Association Frangaise pour l'avancement des Sciences, Congrès de Marseille 1891 ; Mathesis, 1892, pp. 58-64 81-92; Nouvelles Annales de Mathématiques, Janvier 1893, etc; et pour l'application au tétraèdre, Association Francaise, Congrès de Besanron, 1893.

Nous allons donner maintenant quelques exemples des applications de la trangformation continue. Cela nous fournira en méme temps l'occasion d'attirer l'attention sur l'emploi de formules symétriques entre les éléments du triangle, emploi qui est très avantageux pour effectuer beaucoup de calculs qui, à première vue, paraitraient inextricables.

Je me propose, d'abord, de calculer les huit rayons des cercles qui sont tangents aux trois cercles décrits des sommets $\mathrm{A}, \mathrm{B}, \mathrm{C}$ d'un triangle comme centres avec $\mathrm{BC}=a, \mathrm{CA}=b, \mathrm{AB}=c$ comme rayons.

Prenons la question générale de la recherche des rayons des cercles tangents à trois cercles donnés de centres $\mathrm{A}, \mathrm{B}, \mathrm{C}$ et de rayons que j'appelle $R_{b}, R_{b}, R_{c}$. Ce que nous allons faire ainsi est 
une nuanière de traiter le célèbre problèine d'Apollonius, nunière qui, je crois, n'a pas encore été considérée. Pour tixer les idées nous supposons, sur la figure (Fig. 1) qu'il s'agit de chercher le rayon $\rho$ du cercle de centre o qui a les trois cercles donnés à l'extérieur.

Joignons oA, oB, oC que nous appelons $\mathrm{X}, \mathrm{Y}, \mathrm{Z}$; pour tout point du plan on a la relation

$$
\Sigma a^{2} \mathrm{X}^{4} \quad \Sigma\left(b^{2}+c^{2}-a^{2}\right)\left(a^{2} \mathrm{X}^{2}+\mathrm{Y}^{2} Z^{2}\right)+a^{2} b^{2} c^{2}=0
$$

entre les distances $\mathrm{X}, \mathrm{Y}, \mathrm{Z}$ d'un point quelconque aux trois sommets.

D'ailleurs, comme on a :

$$
\mathbf{X}=\mathbf{R}_{w}+\rho, \mathbf{Y}=\mathbf{R}_{b}+\rho, Z=\mathbf{R}_{\mathbf{c}}+\rho
$$

on peut écrire

$\Sigma a^{2}\left(\rho+\mathbf{R}_{a}\right)^{4}-\Sigma\left(b^{2}+c^{2}+c^{2}\right)\left\{c^{2}\left(\rho+\mathbf{R}_{a}\right)^{2}+\left(\rho+\mathbf{R}_{b}\right)^{2}\left(\rho+\mathrm{R}_{c}\right)^{2}\right\}+a^{2} b^{2} c^{2}=0$

Si l'on développe cette équation en l'ordonnant par rapport à $\rho$, on voit très facilement que les coëfficients des termes en $\rho^{4}$ et en $\rho^{\text {; }}$ sont nuls identiquement et il vient

$$
\begin{array}{r}
\rho^{2} \Sigma\left[6 a^{2} \mathbf{R}_{a}{ }^{2}-\left(b^{2}+c^{2}-a^{2}\right)\left\{a^{2}+\left(\mathbf{R}_{b}+\mathbf{R}_{c}\right)^{2}+2 \mathbf{R}_{b} \mathbf{R}_{c}\right\}\right] \\
+2 \rho \Sigma\left[2 a^{2} \mathbf{R}_{a}{ }^{3}-\left(b^{2}+c^{2}-a^{2}\right)\left\{a^{3} \mathbf{R}_{a}+\mathbf{R}_{b} \mathbf{R}_{c}\left(\mathbf{R}_{b}+\mathrm{R}_{c}\right)\right\}\right] \\
+a^{2} b^{2} c^{2}+\sum\left\{a^{2} \mathbf{R}_{a}{ }^{4}-\left(b^{2}+c^{2}-a^{2}\right)\left(a^{2} \mathbf{R}_{a}{ }^{2}+\mathbf{R}_{b}{ }^{2} \mathbf{R}_{c}{ }^{2}\right)\right\}=0
\end{array}
$$

Cette équation donnera deux valeurs $\rho^{\prime}$ et $\rho^{\prime \prime}$ correspondant aux cercles qui touchent les trois cercles donnés en les ayant tous les trois à l'extérieur, ou tous les trois à l'intérieur.

Pour revenir au problème que je me proposais de résoudre, il faut faire :

$$
\mathbf{R}_{\boldsymbol{n}}=a, \mathbf{R}_{b}=b, \mathbf{R}_{c}=c
$$

et calculer le coéfticient de $\rho^{2}$, celui de $\rho$, et le terme indépendant de $\rho$. Appelons $\mathbf{I}, \mathbf{M}, \mathbf{N}$ ces coëfficients, il faut évaluer maintenant

$$
\begin{aligned}
& \mathbf{L}=\Sigma\left[6 a^{4}-\left(b^{2}+c^{2}-a^{2}\right)\left\{a^{2}+(b+c)^{2}+2 b c\right\}\right] \\
& \mathbf{M}=\Sigma\left[2 a^{5}-\left(b^{2}+c^{2}-a^{2}\right)\left\{a^{3}+b c(b+c)\right\}\right] \\
& \mathbf{N}=a^{2} b^{2} c^{2}+\Sigma\left\{a^{6}-\left(b^{2}+c^{2}-a^{2}\right)\left(a^{4}+b^{2} c^{2}\right)\right\} .
\end{aligned}
$$


Calcul de L. On peut écrire

$$
\begin{aligned}
& \mathrm{L}=6 \mathrm{\Sigma} a^{4}-\Sigma\left(a^{2}+b^{2}+c^{2}-2 a^{2}\right)\left(a^{2}+b^{2}+c^{2}+4 b c\right), \text { ou } \\
& \mathrm{L}=6 \Sigma a^{4}-\Sigma\left(a^{2}+b^{2}+c^{2}\right)^{2}+2 \Sigma a^{2}\left(a^{2}+b^{2}+c^{2}\right)-4 \Sigma b c\left(a^{2}+b^{2}+c^{2}\right) \\
& +8 \Sigma a^{2} b c \text {, ou } \\
& \mathrm{L}=6 \mathbf{U}^{4}-3\left(a^{2}+b^{2}+c^{2}\right)^{2}+2\left(a^{2}+b^{2}+c^{2}\right) \mathbf{2}^{2}-4\left(c^{2}+b^{2}+c^{2}\right) \mathrm{S} w \\
& +8 a h c=\text { 'r, ou } \\
& \mathrm{L}=6 \Sigma a^{4}-2\left(a^{2}+b^{2}+c^{2}\right)^{2}-4\left(a^{2}+b^{2}+c^{2}\right) \Sigma b c+16 p a b c .
\end{aligned}
$$

Mais les formules dont je parlais tout à l'heure (voir Mathesis 1892, loco citato) donnent

$$
\begin{gathered}
\left.\Sigma a^{4}=2\left\{p^{2}-r \hat{0}\right)^{2}-4 \mathrm{~S}^{2}\right\}, \quad a^{2}+b^{2}+c^{2}=2\left(p^{2}-r \delta\right) \\
\Sigma b r=p^{2}+\gamma^{2} \delta:
\end{gathered}
$$

on a d'ailleurs, $\quad \quad \quad \quad\left(r=4\right.$ Rs et $\dot{s}=\mu^{m}$.

Substituant et effectuant les calculs, on trouve tris facilement

$$
\left.\mathrm{L}=16, r^{\circ}(\not \ddot{*}-4)^{2}\right)
$$

Calcul de $\mathrm{M}$. On $n$ :

$$
\begin{aligned}
& \mathbf{M}=2 \Sigma a^{5}-\Sigma\left(b^{2}+c^{2}-a^{2}\right) a^{3}-\Sigma b c\left(b+c^{\prime}\right)\left(b^{2}+c^{2}-a^{2}\right), \text { ou } \\
& \mathbf{M}=2 \Sigma a^{5}-\Sigma\left(a^{2}+b^{2}+c^{2}-2 a^{2}\right) a^{3}-\Sigma b c\left(b+c^{2}\right)\left(a^{2}+b^{2}+c^{2}-2 a^{2}\right), \text { ou } \\
& \mathbf{M}=4 \Sigma a^{5}-\left(a^{2}+b^{2}+c^{2}\right) \Sigma a^{3}-\left(a^{2}+b^{2}+c^{2}\right) \Sigma b c(b+c)-2 a b r \Sigma(b+c) n, \text { ou } \\
& \mathbf{M}=4 \Sigma a^{5}-\left(a^{2}+b^{2}+c^{2}\right) \Sigma b^{3}-\left(a^{2}+b^{2}+c^{2}\right) \Sigma b c(a+b+c-a) 4 a b c \Sigma b c, \text { ou } \\
& \mathbf{M}=4 \Sigma a^{5}-\left(a^{2}+b^{2}+c^{2}\right) \Sigma a^{3}-2 p\left(a^{2}+b^{2}+c^{2}\right) \Sigma b c+3 a b c\left(a^{2}+b^{2}+c^{2}\right) \\
& -4 a b c b c
\end{aligned}
$$

Les formules déjà citées donnent

$$
\begin{aligned}
& \Sigma a^{3}=2 p\left(p^{2}+6 \mathrm{R} r-3 r \delta\right)=2 p\left\{p^{2}-3 r(2 \mathrm{R}+r)\right\} \\
& \Sigma a^{5}=2 p\left(p^{4}-10 p^{2} r(\mathrm{R}+r)+5 r^{2} \delta(2 \mathrm{R}+r)\right\}
\end{aligned}
$$

Substituant et réduisant, on trouve

$$
\mathbf{M}=64 p r^{2}\left\{\delta(2 \mathrm{R}+r)-2 p^{2}\right\}
$$


Calcul de N. On a

$$
\begin{aligned}
& \mathrm{N}=a^{2} b^{2} c^{2}+\sum\left\{a^{6}-\left(b^{2}+c^{2}-a^{2}\right)\left(a^{4}+b^{2} c^{2}\right)\right\} \quad \text { ou } \\
& \mathrm{N}=a^{2} b^{2} c^{2}+\Sigma a^{6}-\Sigma\left(a^{2}+b^{2}+c^{2}-2 a^{2}\right)\left(a^{4}+b^{2} c^{2}\right) \text { ou }
\end{aligned}
$$

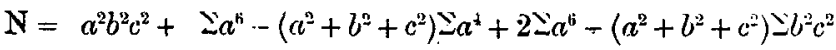

$$
\begin{aligned}
& +2 a^{2} b^{2} c^{2} \text { ou } \\
& \mathbf{N}=7 a^{2} b^{2} c^{2}+3 a^{6}-\left(a^{2}+b^{2}+c^{2}\right)^{2} a^{4}-\left(a^{2}+b^{2}+c^{2}\right) \Sigma b^{2} c^{2}
\end{aligned}
$$

On trouve daus nos formules

$$
\Xi b^{\prime \prime} c^{\prime \prime}=\left(p^{2}-r \delta\right)^{2}+4 S^{\prime \prime}
$$

On n'y trouve pas $\triangleq a^{6}$ mais il peut se calculer aisément en partant de ${ }^{2} a^{+}$et de ${ }^{2} a^{2}$.

On a en effet

$$
\begin{aligned}
& \left(a^{4}+b^{4}+c^{4}\right)\left(a^{2}+b^{2}+c^{2}\right)=2 a^{6}+2 a^{2}\left(b^{4}+c^{4}\right) \\
& =\mathrm{L}^{\mathrm{b}}+\mathrm{v}^{2} b^{2} c^{2}\left(b^{2}+c^{2}\right) \text { ou }
\end{aligned}
$$$$
4\left\{\left(p^{2}-r \cdot \delta\right)^{2}-4 S^{2}\right\}\left(p^{2}-r \cdot \delta\right)=\Sigma a^{6}+2 b^{2} \cdot c^{2}\left(a^{2}+b^{2}+c^{2}-a^{2}\right)
$$$$
=\Sigma a^{6}+2\left(p^{2}-\imath \delta\right) \Sigma b^{2} c^{2}-3 a^{2} b^{2} c^{2} \text { ou }
$$

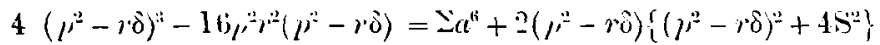

$$
-48 p^{*} \mathbf{K}^{2} r^{2}
$$

d'ou

$$
\Sigma \alpha^{6}=2\left(\gamma^{2}-r \delta\right)^{3}-24 p^{2} \gamma^{2}\left(p^{2}-r \delta\right)+48 p^{2} \mathrm{R}^{2} v^{2}
$$

Substituant on trouve

$$
\left.\mathrm{N}=6+p^{2} r^{2}\left\{(2 \mathrm{R}+r)^{2}-p^{2}\right)^{2}\right\}
$$

Remarquons, en passant, que l'on a

$$
r^{2}-(2 \mathrm{R}+r)^{2}=4 \mathrm{R}^{2} \cos \mathrm{A} \cos \mathrm{B} \cos \mathrm{C}
$$

L’équation qui déternine $\rho$ devient alors

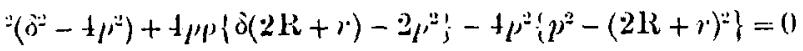

2 Vol. 13 
On en tire, toutes réductions faites :

$$
\rho^{\prime}=\frac{2 \mu(2 \mathrm{R}+r-p)}{2 p-\delta} \quad \rho^{\prime \prime}=-\frac{2 p(2 \mathrm{R}+r+p)}{2 p+\delta}
$$

La formule (1) lorsque les racines sont réelles donne toujours les rayons avec un signe, naturellement; mais il faut interpréter géométriquement ce signe. Ainsi, avec les deux formules précédentrs, si le triangle est équilatéral, on trouve

$$
\rho^{\prime}=-\frac{\sqrt{3}-1}{\sqrt{3}}, \quad \rho^{\prime \prime}=-\frac{\sqrt{3}+1}{\sqrt{3}}
$$

et ce sont bien, mais en valeur absolue seulement, les valeurs des rayons qui conviennent pour ce cas, comme la géométrie le montre immédiatement. (Voir la note additionnelle à la tin du mémoire.)

Si l'on applique la T'ransformation continue en $\mathbf{A}$ aux formules $(2)$, il vient

$$
\begin{aligned}
& \rho_{a}^{\prime}=\frac{2(p-a)\left\{-2 \mathbf{R}+r_{a}+(p-a)\right\}}{2(p-a)-\delta_{a}}, \\
& \rho_{a}^{\prime \prime}=-\frac{2(p-a)\left\{-2 \mathbf{R}+r_{a}+(p-a)\right\}}{2(p-a)+\delta_{a}}
\end{aligned}
$$

qui donnent les rayons du couple de cercles tangents aux trois cercles donnés, le premier tangent à l'intérieur du cercle de centre $A$ et à l'extérieur des deux autres, le second tangent à l'extérieur du cercle de centre $A$ et à l'interieur des deux autres; on aurait de même les rayons des cercles des deux autres couples par transformation continue en B et en C.

Ces calculs paraissent fort longs surtout parceque nous les avons développés, dans le but de montrer, pour des cas analogues, notre manière d'opérer, mais ils sont très symétriques, très aisés, et nous ne savons d'ailleurs pas comment on aurait pu arriver à ces résultats sans nos formules et sans la I'ransformation continue; il ne serait probablement même point facile d'y arriver synthétiquement en supposant ces résultats connus. et en cherchant alors à les démontrer. 


\section{REMARQues.}

L'axe de similitude externe des trois circonférences

$$
\mathbf{A}(a), \mathbf{B}(b), \mathbf{C}(c) \text { est la droite } c^{2} x+b^{2} y+c^{2} z=0 .
$$

On en conclut, par transformation continue en $\mathrm{A}$, que la droite (coordonnées normales) $-a^{2} x+b^{2} y+c^{2} z=0$ est l'axe de similitude qui passe par les centres de similitude interne de $\mathrm{A}(a)$ et $\mathrm{B}(b)$, et de $\mathbf{A}(a)$ et $\mathrm{C}(c)$. Nous désignons par $\mathrm{M}(\mathrm{R})$ un cercle de rayon $\mathrm{R}$ et de centre $M$.

Le centre radical de ces trois circonférences est le point dont les coordonnées normales sont:

$$
\cos A-\cos B \cos C, \cos B-\cos C \cos A, \cos C-\cos A \cos B
$$

c'est un point que l'on rencontre assez souvent dans la Géométrie du triangle et qui est le symétrique de l'orthocentre par rapport au centre du cercle circonscrit.

On trouve très simplement les ccordonnées de ce centre radical; en effet (Association française, 1888, Congrès d'Oran, p. 170 vi.) il a pour coordonnées normales

$$
a b c \cos \mathrm{A}-a^{3}+b^{3} \cos \mathrm{C}+c^{3} \cos \mathrm{B} \text {, etc. }
$$

Mais $\quad b^{3} \cos \mathrm{C}+c^{3} \cos \mathrm{B}-a^{3 \prime}=4 \mathrm{RS}(\cos \mathrm{A}-2 \cos \mathrm{B} \cos \mathrm{C})$;

elles deviennent donc

$$
4 R S \cos A+4 R S(\cos A-2 \cos B \cos C) \text {, etc. }
$$

ou $\cos \mathrm{A}-\cos \mathrm{B} \cos \mathrm{C}$, comme nous l'avons dit.

La remarque permet de placer très simplement ce point dans le triangle ABC.

Pour cela je trace les trois cercles $\mathrm{A}(a), \mathrm{B}(b), \mathrm{C}(c)$ op : $\left(9 \mathrm{C}_{1}+3 \mathrm{C}_{3}\right)$ et comme ces cercles se rencontrent deux à deux, il suffit de tracer dev $x$ de leurs intersections qui se coupent au point cherché.

$$
\text { En tout op: }\left(4 R_{1}+2 R_{2}+9 C_{1}+3 C_{33}\right)
$$$$
\text { op : }\left(\mathbf{4} \mathbf{R}_{1}+2 \mathbf{R}_{2}\right)
$$

Simplicité 18 ; exactitude $13 ; 2$ droites, 3 cercles. 
Sans entrer dans d'autres détails j'énoncerai encore les applications suivantes de la transformation continue et de nos formules.

Les points $\omega, \omega^{\prime}$ qui ont pour coordonnées normales respectivement

$$
\frac{a+r_{a}}{a}, \frac{b+r_{b}}{b}, \frac{c+r_{r}}{c} ; \quad \frac{a-r_{a}}{a}, \frac{b-r_{b}}{b}, \frac{c-r_{c}}{c}
$$

sont des points qui jouissent de propriétés remarquables et que j'ai souvent rencontrés, ainsi que leurs transformés continus en $A: \omega_{\triangleleft}, \omega_{\triangleleft}{ }^{\prime}$

$$
\frac{a+r}{a}, \frac{b+r_{c}}{b}, \frac{c+r_{b}}{c} ; \quad \frac{a-r}{a}, \frac{b-r_{c}}{b}, \frac{c-r_{b}}{c}
$$

en $B: \omega_{b}, \omega_{b}^{\prime}$, etc.

Ces huit points $\omega, \omega^{\prime}, \omega_{\omega}, \omega_{a}^{\prime}$, etc., sont les centres des quatre couples de cercles tangents savoir :

$\omega, \omega^{\prime}$ aux cercles $\mathrm{A}(p-a), \mathrm{B}(p-b), \mathrm{C}(p-c)$ tangents deux à deux ;

$\omega_{a,} \omega_{a}^{\prime} \quad$ aux cercles $\mathrm{A}(p), \mathrm{B}(p-c), \mathrm{C}(p-a)$;

$\omega_{\iota}, \omega_{b}^{\prime}$ etc.

On peut trouver les rayons de ces cercles en appliquant la formule générale que nous avons donnée plus haut pour résoudre le problème d'Apollonius. Il suffit de faire :

pour $\quad \omega$ et $\omega^{\prime}, \mathbf{R}_{a}=p-a, \mathbf{R}_{b}=p-b, \mathbf{R}_{c}=p-c$

pour $\omega_{n}$ et $\omega_{c}^{\prime}, \mathrm{R}_{\iota}=p \quad, \mathrm{R}_{b}=p-c, \mathrm{R}_{c}=p-b$

et de réduire les coetticients de l'équation en $\rho^{2}$ par une méthode analogue à celle que nous avons donnée. On trouvera pour les cercles de centres $\omega$ et $\omega^{\prime}$

$$
\rho=\frac{\mathrm{S}}{2 p+\delta}, \quad \rho^{\prime}=\frac{\mathrm{S}}{2 p-\delta}, \text { etc. }
$$


On a :

$$
\overrightarrow{\omega \omega^{\prime 2}}=16 \mathrm{~S}^{2} \frac{\delta^{2}-3 p^{2}}{\left(4 p^{2}-\delta^{2}\right)^{2}}
$$

et par transformation continue en $\mathrm{A}$

$$
\overline{\omega_{a} \omega_{a}{ }^{2}}=16 \mathrm{~S}^{2} \frac{\delta_{a}{ }^{2}-3(p-a)^{2}}{\left\{4(p-a)^{2}-\delta_{n}{ }^{2}\right\}^{2}}
$$

Les coordonnées cartésiennes du centre $O$ du cercle inscrit par rapport à $H B$ pris pour axe des $x$ et à $H A$ pour axe des $y$ sont, comme il est facile de le voir, (H étant l'orthocentre)

$$
x=\frac{c \cos \mathrm{B}-(p-b)}{\sin \mathrm{C}} \quad y=\frac{r \sin \mathrm{C}-(p-b) \cos \mathrm{C}}{\sin \mathrm{C}}
$$

En appliquant la transformation continue en $\mathbf{A}$, en $\mathrm{B}$, en $\mathrm{C}$ à ces expressions de coordonnées on a imncédiatement les coordonnées, par rapport à ces mêmes axes, des centres $\mathrm{O}_{a}, \mathrm{O}_{b}, \mathrm{O}_{c}$, des cercles exinscrits.

$$
\begin{array}{ll}
\mathrm{O}_{n}: & x=\frac{c \cos \mathrm{B}-(p-c)}{\sin \mathrm{C}}, \quad y=-\frac{r_{n} \sin \mathrm{C}+(p-c) \cos \mathrm{C}}{\sin \mathrm{C}} \\
\mathrm{O}_{b}: & x=\frac{c \cos \mathrm{B}-p}{\sin \mathrm{C}}, y=\frac{r_{h} \sin \mathrm{C}-p \cos \mathrm{C}}{\sin \mathrm{C}} \\
\mathrm{O}_{c}: & x=\frac{\cos \mathrm{B}+(p-a)}{\sin \mathrm{C}}, y=\frac{r \sin \mathrm{C}+(p-a) \cos \mathrm{C}}{\sin \mathrm{C}}
\end{array}
$$

L'équation, en coordonnées normales, de l'ellipse qui a pour foyers deux sommets du triangle, $\mathbf{B}$ et $\mathbf{C}$ par exemple, et passe par le troisième en $A$, est

$$
p(p-a)\left(b^{2} y^{2}+c^{2} z^{2}\right)+b c y \approx\left\{p^{2}+(p-a)^{2}\right\}+a b c x \cdot(b+c)(y+z)=0
$$

Si on la transforme en $A$, elle se reproduit, mais si on la trans. forme soit en $B$, soit en $C$, on obtient l'équation de l'hyperbole qui a pour foyers $B$ et $C$ et passe en $A$

$(p-b)(p-c)\left(b^{2} y^{2}+c^{2} z^{2}\right)-b c y z\left\{(p-b)^{2}+(p-c)^{2}\right\}+a b c x(b-c)(y-z)=0$ 
Je vais donner quelques explications sur la façon dont j'ai obtenu les nombreuses formules auxquelles je fais souvent allusion ici, et que j'ai employées, sans les démontrer; elles dérivent des formules connues

$$
\begin{aligned}
\mathrm{S} & =p r, \text { etc., } r_{a}+r_{b}+r_{r}=4 \mathrm{R}+r, \\
p(p-a) & =r_{b} r_{c},(p-b)(p-c)=r r_{a}, \text { etc. }
\end{aligned}
$$

et de quelques autres que j'ai rencontrées, et qui ne l'étaient pas ou du moins dont on n'avait pas remarqué la fécondité. Je citerai par exemple les trois suivantes

$$
\begin{aligned}
\cos \mathbf{A} & =\frac{2 \mathrm{R}+r-r_{n}}{2 \overline{\mathrm{R}}} \\
a^{2}+b^{2}+c^{2} & =2\left(p^{2}-r \delta\right) \\
b c+c a+a b & =p^{2}+r \delta
\end{aligned}
$$

La première peut se démontrer ainsi.

Soient $x, y, z$ les perpendiculaires abaissées du centre du cercle circonscrit sur les trois côtés, on a :

$$
x+y+z=\mathbf{R}+r
$$

C'est un théorème de Carnot dont M. J. S. Mackay a donné de nombreuses démonstrations dans son intéressant mémoire The Triangle and its Six Scribed Circles (Edinburgh Mathematical Society, 1883).

$\mathrm{Si}$ nous transformons l'équation (1) continûment en $\mathbf{A}$ elle devient

$$
-x+y+z=-\mathbf{R}+r_{n}
$$

De (1) et de (2) on déduit

$$
x=\frac{2 \mathrm{R}+r-r_{\text {u }}}{2}
$$

Mais

$$
\begin{aligned}
x & =\mathrm{R} \cos \mathrm{A} \quad ; \text { donc } \\
\cos \mathrm{A} & =\frac{2 \mathrm{R}+r-r_{n}}{2 \mathrm{R}} .
\end{aligned}
$$

Je ne vois pas de moyen de démontrer les deux autres formules par des considérations géométriques simples sur une figure, 
et ce serait à désirer, mais voici la façon de les obtenir ensemble. On a

$$
\begin{aligned}
r_{l}+r_{b}+r_{c}=4 \mathrm{R}+r & =\delta \\
\text { d'où } \quad \frac{1}{b+c-a}+\frac{1}{c+a-b}+\frac{1}{u+b-r} & =\frac{2 \mathrm{~S}}{\delta}
\end{aligned}
$$

puisque $\mathrm{S}=r_{a}(p-a)$, etc.

De là je tire

$$
\begin{aligned}
\frac{a^{2}-(b-c)^{2}+b^{2}-(c-a)^{2}+c^{2}-(a-b)^{2}}{16 \mathrm{~S}^{2}} & =\frac{4 \mathrm{~S} p}{\delta} \\
2 \Sigma b c-\check{\Sigma} a^{2} & =4 \delta r
\end{aligned}
$$

d'où

Mais on a identiquement

$$
2 \searrow b c+\Sigma a \quad=4 p
$$

d'où l'on tire

$$
\Sigma b c=p^{2}+r \delta \quad \text { et } \Sigma u^{2}=2\left(p^{2}-r \delta\right)
$$

Je bornerai là ce que je veux dire de ces formules, mis puisque c'est M. J. S. Mackay qui me fait l'honneur de présenter cette note à la Société Mathématique d'Edinburgh, je veux aussi injouter quelques observations relatives à la Géométrographie qu'il vous a fait connaître, il y a quelques mois, en l'appliquant devant vous à la recherche du symbole, de la simplicité et de l'exactitude des constructions données dans l'Euclide, employé presque universellement, en Angleterre, pour l'étude des éléments de Géométrie.

Nous sommes sur le sujet, tout à fait d'accord, nous ne différons que sur des détails très peu importants au fond; seulement en dehors de l'avantage sérieux d'avoir partout identiquement les mêmes notations, je pense que celles que j'emploie, sont plus dans l'esprit de la méthode telle que je l'ai conçue, et je vais essayer brièvement de le convaincre.

Je rappelle que l'essence de la Géométrographie est spéculative, elle ne s'applique aux constructions à effectuer que-si parva licet componere magnis-comme la Mécanique rationnelle s'applique à l'art de l'Ingénieur. Voici mes notations 
1. Faire passer le bord d'une règle par un point placé, c'est l'opération

donc, spéculativement, faire passer le bord d'une règle par deux points placés, c'est op : $\left(2 R_{1}\right)$

2. Tracer la droite qui suit le bord d'une règle, c'est op : $\left(R_{2}\right)$

3. Mettre une pointe d'un compas en un point placé, c'est

op : $\left(\mathrm{C}_{1}\right)$

donc, spéculativement, prendre avec le compas une longueur placée, c'est

op : $\left(2 \mathrm{C}_{1}\right)$

4. Mettre une pointe en un point indéterminé d'une ligne tracée, c'est

5. Tracer le cercle, c'est

op : $\left(\mathrm{C}_{3}\right)$

Monsieur Mackay supprime l'opération $\mathrm{C}_{2}$ qu'il assimile à $\mathrm{C}_{1}$, parceque, dit-il, quand on met la pointe en un point indéterminé d'une ligne on vise d'abord le point où l'on veut placer la pointe, c'est à dire qu'on la met réellement en un point déterminé; entin l'on a ainsi l'avantage d'avoir plus de symétrie dans les symboles, puisque, supprimant mon symbole $C_{2}$, il appelle $C_{2}$ ce que j'appelle $C_{3}$ et que l'on a: $R_{1}, R_{2}$ symboles pour la droite, $C_{1}, C_{2}$ symboles pour le cercle.

Monsieur Mackay a raison, l'on vise effectivement, en pratique, un point avant d'y poser la pointe, mais peu inporte; au point de vue spéculatif de la Géométrographie, mettre une pointe en un point déterminé et mettre une pointe en un point indéterminé d'une ligne, sont deux choses essentiellement différentes; c'est pour cela que je crois préférable de conserver les notations $\mathrm{C}_{1}$ et $\mathrm{C}_{2}$ atin de marquer la différence, puisque, d'ailleurs, il n'y a aucun inconvénient à la chose. Quant à l'avantage de la symétrie, il serait grand, si l'on avait à faire des calculs avec les symboles comme avec les expressions algébriques, mais comme le symbole final est sirnplement un tableau qui ne sert qu'à lire des résultats, il suffit qu'il soit clair et la suppression du symbole $C_{2}$ n'y apporte, pas plus de clarté et fait disparaître un détail des phases de l'opération. La symétrie pour l'oeíl, à un point de vue en quelque sorte esthétique, n'est dans ce cas qu'une illusion, car elle ne s'appliquerait qu'à une partie de la 
Géométrographie, celle qui étudie les constructions canoniques de la règle et du compas, elle disparaîtrait avec l'adjonction des symboles nouveaux qu'amène l'emploi de l'équerre, ainsi que je l'ai développé dans un mémoire présenté au mois d'Août cette année au Congrès de l'Association Française, à Caen. Ajoutons que la question reste, en réalité, presque du domaine de la théorie, car le symbole $\mathrm{C}_{2}$ se rencontre assez rarement dans les constructions et généralement par faibles unités dans celles où il se rencontre.

Enfin nous avons apprécié différemment dans un cas-très peu important également-la manière de compter les symboles.

Pour tracer deux droites $\mathrm{AB}, \mathrm{AC}$ passant par un même point, je ne tiens pas compte de ce qu'elles passent par le même point et j'évalue le tracé $4 R_{1}+2 R_{2}$ comme s'il s'agissait de deux droites différentes Al, CD. Cela, par la raison qu'on ne peut maintenir la règle en $A$ (une fois qu'on a tracé $A B$ ). Pour tracer $A C$ on recommence simplement l'opération sans que la nouvelle opération profite en quoique ce soit de ce qu'on a fait pour la première. Monsieur Mackay compte $2 R_{1}+R_{2}$ pour la première et seulement $R_{1}+R_{2}$ pour la seconde, comme si la règle avait tourné autour de A. Je m'aperçois très bien que je m'appuie sur deux ordres de raisons qui semblent se contredire; en effet pour justifier l'emploi de $C_{2}$, je m'appuie sur l'essence spéculative de la Géométrographie et, pour justifier mon évaluation du tracé successif de deux droites qui ont un point commun, j'invoque la manœurre pratique que l'on exécute. Cela est fort naturel cependant, parceque, quoique spéculative, la Géométrographie a en vue l'application possible de ses spéculations, elle a donc deux faces et, si elle doit rester tout à fait spéculative lorsque cela n'a aucun inconvénient,-_c'est ainsi que pour tracer le cercle $O(b)$ immédiatement après avoir tracé le cercle $O(a)$, je ne conpte que $C_{3}$, si je n'ai pas eu à déplacer la pointe fixée en $O$, mais seulement à ouvrir ou à fermer la branche du compas, parceque je puis opérer ainsi-il me semble utile de conclescendre à la pratique dans le cas où j'ai à tracer deux droites successives ayant un point commun puisque le contraire aurait un inconvénient et que je suis, de plus près, la manouvre du tracé.

Je ne m'étendrai pas sur l'emploi que j'ai fait de l'équerre en appliquant la Géométrographie à la Géonétrie descriptive, je renvoie pour cela à mon mémoire déjà cité, présenté au Congrès de Caen. Je vais seulement définir les nouveaux symboles que j’ai adoptés en les ajoutant aux anciens. 
Je suppose que l'on emploie l'équerre seulement pour tracer les parallèles et jamais pour tracer les perpendiculaires; je ne suis point habitué à la pratique du dessin, mais je sais que cette manière d'opérer est proscrite dans toutes les épures exactes. Par exemple les instructions données aux dessinateurs des services de la Ville de Palis recommandent de ne jamais tracer de perpendiculaires avec l'équerre; si l'on a plusieurs perpendiculaires à tracer à une même direction, on détermine la première avec la règle et le compas et les autres avec l'équerre, comme étant parallèles à celle-ci.

Nous admettons cependant que l'on se serve d'un té dont la direction donne les parallèles à la ligne de terre sur la feuille collée sur la planche, et alors, que l'on puisse mener, à l'équerre, les lignes de rappel relatives à cette ligne de terre, après avoir vérifié l'exactitude du tracé une fois pour toutes.

On n'a donc, pour l'usage de l'équerre, que les opérations nou velles suivantes (sans compter celles où l'équerre sert comme servirait la règle seule, opérations qui conservent le symbole adopté).

-Mettre an bord de la règle ou de l'équerre en coincidence avec une droite déjù tracé sur la figure. Nous pourrions assimiler cette opération à faire passer le bord par deux points et compter alors $2 R_{1}$, naais il nous semble utile-et sans aucun inconvénient-de distinguer, légèrement, cette nouvelle opération, afin de pouvoir apprécier, d'un coup d'oeil jeté sur le symbole final de la construction, le degré de fréquence dans l'emploi qu'on y a fait de l'équerre ; aussi nous l'appellerons

op : $\left(2 \mathbf{R}_{1}^{\prime}\right)$

Nous ne comptons pas l'acte tout mécanique de placer l'équerre contre la règle ou réciproquement.

-Faire glisser l'équerre jusqu' à ce que son bord passe en un point placé

$R_{1}^{\prime}$ et $E$ sont les seuls symboles nouveaux à employer et, toute opération faite avec le compas, la règle et l'équerre, aura pour symbole :

$$
\begin{aligned}
\mathrm{Op}: & \left(l_{1} \mathrm{R}_{1}+l_{2} \mathrm{R}_{1}^{\prime}+l_{3} \mathrm{R}_{2}+l_{4} \mathrm{E}+l_{5} \mathrm{C}_{1}+l_{6} \mathrm{C}_{2}+l_{7} \mathrm{C}_{3}\right) \\
& l_{1}+l_{2}+\ldots+l_{7} \text { sera le coëfficient de simplicité } \\
& l_{1}+l_{2}+l_{4}+l_{5}+l_{6} \text { le coëfficient d'exactitude } \\
& l_{;} \text {sera le nombre de droites tracées } \\
& l_{7} \text { celui des cercles. }
\end{aligned}
$$


Je veux terminer en vous donnant les résultats d'une expérience que la complaisance de M. Jung, le savant professeur de Milan, m'a permis d'exécuter et qui montre jusqu' où peut aller la simplification que la Géométrographie introduit dans une construction, simplification qui est due surtout à l'idée fondamentale de la Géométrographie, c'est à dire à la recherche méthodique des simplifications; car la Géométrographie n'innove rien en Géométrie, elle se sert d'éléments connus, et, à la rigueur, toutes les simplifications auxquelles elle conduit, auraient pu théoriquement être faites sans elle : mais on n'y songeait pas, parceque l'on manquait de criterium et parceque le mot de simplicité ne s'appliquait qu'à l'exposition théorique de la solution d'un problème et non à sa construction effectuée.

Voici l'expérience dont il s'agit. Je voulais prier un géomètre, tout à fait étranger à la notion de Géométrographie, de m'écrire en détail, sans faire l'épure, comment il s'y prendrait pour exécuter, avec la règle et le compas, le plus simplement qu'il le pourrait, selon les règles connues de la construction des expressions algébriques, une construction quelconque que je lui indiquerais au hasard. D'après cela je pourrais évaluer sa construction par le symbole géométrographique, puis reprendre, moi, le même problème en y ipportant les simplitications méthodiques de la Géométrographie; enfin évaluer ma construction et comparer les deux symboles. Je ne pouvais faire facilement la chose à Paris car les mathématiciens qui y sont de mes relations connaissent, au moins vaguement, la Géométrographie, et je n'aurai pas eu la construction dans les conditions qu'il me fallait. M. Jung avec qui j'ai le plaisir d'être en rapport, précisement à propos de Géométrographie, accepta de m'aider à faire l'expérience. Je lui envoyai alors cette construction :

Dans un triangle $A B C$ dont les côtés sont $a, b, c$ placer le puint dont les distances uua trois côtés $B C, C A, A B$, (ou coordonnées normales), sont respectivement proportionelles $\grave{a}$ :

$$
\frac{a^{2} b^{2}+a^{2} c^{2}-b^{2} c^{2}}{a}, \quad \frac{b^{2} c^{2}+b^{2} a^{2}-a^{2} c^{2}}{b}, \quad \frac{c^{2} a^{2}+c^{2} b^{2}-a^{2} b^{2}}{c}
$$

c'est un point que je choisis au hasard parmi ceux qui se rencontrent fréquemment dans la Géométrie du triangle avec des coordonnées un peu coniplexes. 
Il était convenu que le point devait être placé directement d'après ses coordonnées et non d'après des propriétés géométriques particulières à ce point, si l'on en trouvait. Monsieur Jung pria un de ses anciens élèves de faire ce que je désirais, et quelques jours après il eut la bonté de m'envoyer la rédaction même qui lui avait été remise.

Je dois dire que la construction qu'on y indique est très logique, fort bien conçue et analogue à celles que chacun de vous ferait sans doute, et que moi-même j'eusse fait il y a quelques années. On en trouverait, facilement, sans Géométrographie, de beaucoup plus simples à tracer, mais il n'y aurait aucun criteriunı pour permettre de l'affirmer, et cette préoccupation du tracé réel n'est pas dans l'esprit des Géomètres.

Je déterminai alors le symbole de cette construction que je conduisis même assez économiquement en évitant certaines répétitions de lignes inutiles. Puis je cherchai une construction de ce mème point en appliquant les procédés géométrographiques et j'en déterminai aussi le symbole. Les résultats sont stupéfiants; les voici :

Symbole de la construction qui m'a été envoyée

$$
\text { op : }\left(81 \mathrm{R}_{1}+46 \mathrm{R}_{2}+211 \mathrm{C}_{1}+121 \mathrm{C}_{3}\right)
$$

Simplicité 459 ; exactitude $292 ; 46$ droites, 121 cercles.

Symbole de la construction géométrographique

$$
\text { op : }\left(1+\mathrm{R}_{1}+7 \mathrm{R}_{2}+48 \mathrm{C}_{1}+22 \mathrm{C}_{\mathrm{i}}\right)
$$

Simplicité 91 ; exactitude $62 ; 7$ droites, 22 cercles.

La première exige donc plus de 5 fois plus d'opérations élémentaires et le tracé de 6 fois $\frac{1}{2}$ plus de droites, de 5 fois $\frac{1}{2}$ plus de cercles!

Ce n'est pas tout, un géomètre qui s'est épris de la Géométrographie, s'y est exercé, (et y a même acquis beaucoup plus de dextérité que moi) Monsieur Bernès, auquel j'avais envoyé le problème, parceque je ne doutais pas qu'il n'en simplifiât encore la construction, m'écrit ce matin qu'il l'exécute avec 64 opérations élé. mentaires et qu'il me clonnera la construction à son retour de voyage! Plus de 7 fois plus simple que la construction faite en employant la méthode résultant de ce qui est indiqué dans tous les Traités classiques, wu sujet des constructions. J'avoue que j'ai été moi-même un peu 
surpris de cette invraisemblable différence, je pensais bien que je simplifierais d'au moins la moitié, mais de $j$ fois, de 7 fois, je ne pouvais m'y attendre, d'autant plus que lorsque j'essayais moi-même d'exécuter les constructions d'application par les méthodes usuelles, pour faire la comparaison avec les méthodes géométrographiques, je les simplitiais malgré moi, pour éviter de les compliquer inutilement, et je n'arrivais pas alors à des résultats aussi éloignés dans les constructions traitées par moi avec les deux méthodes; en m'adressant à un géomètre quelconque, j'il évité cette cause d'incertitude, et, sans croire que toutes les constructions d'application-je ne parle pas des constructions fondamentales séculaires, que j'ai cependant presque tontes simplifiées peu ou beaucoup depuis : mener par un point $\mathrm{A}$ une droite parallèle à une droite donnée $\mathrm{BC}-$ donnernient lieu à une aussi considérable réduction, j'estime que la construction géométrographique serait toujours de 2 a 3 fois plus simple que la construction exécutée par les méthodes employées jusquuici.

Paris, Octobre 1894.

NOTE. ADDITIONELLE.

Si l'on convient que les longueurs doivent être comptées positivement à partir de $o$ vers $o A$, on voit que $A_{1}$ est égal à $-\mathbf{R}_{n}$, l'origine des rayons étant en $A$. Les équations de la page 10 doivent donc être $X=\rho-\mathbf{R}_{a}$, etc. Le signe du coefficient de $\rho$ dans l'équation (1) sera simplement changé, et l'on aura les valeurs de $\rho$ avec le signe qui leur convient. 